INRA Prod. Anim., 2004, 17 (3), 243-252

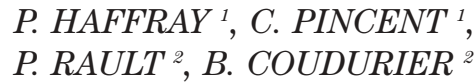

${ }^{1}$ SYSAAF, Station SCRIBE, INRA, Campus de Beaulieu, F-35042 Rennes

${ }^{2}$ SYSAAF, Station de Recherches Avicoles, INRA, F-37380 Nouzilly

Courriel : haffray@beaulieu.rennes.inra.fr

\section{Domestication et amélioration génétique des cheptels piscicoles français dans le cadre du SYSAAF}

La domestication recouvre l'ensemble des modifications génétiques inhérentes au choix des reproducteurs lors du renouvellement des générations. Si une part importante des modifications correspond à la sélection volontaire sur des critères choisis, une part non négligeable semble pouvoir être attribuée à la sélection naturelle qui s'effectue par le simple fait de retenir comme reproducteurs des animaux ayant survécu au processus de l'élevage. Ceux-ci sont généralement moins sensibles à ce changement d'environnement ou à certains agents pathogènes, plus aptes à utiliser certains nutriments ou à se reproduire en condition d'élevage, etc. De nombreux auteurs ont déjà abordé l'impact de la domestication chez les poissons (pour revue : Vandeputte et Prunet 2002).

Les reproducteurs des espèces piscicoles ont été gérés et sélectionnés de façon plus empirique que ceux des espèces terrestres. Les pisciculteurs doivent relever le défi de la domestication simultanée de plusieurs espèces, tout en intégrant des problématiques de rentabilité économique, de préservation de

\section{Résumé}

Depuis 1991, des entreprises françaises sélectionnent la truite arc-en-ciel, la truite fario, le bar, la daurade et le turbot. Les éleveurs ont développé une expertise collective en matière de génétique quantitative, de contrôle de parenté assisté par empreintes génétiques, de monosexage, d'induction hormonale de la ponte, de triploïdisation et de conservation et congélation des gamètes, dans le cadre du Syndicat des Sélectionneurs Avicoles et Aquacoles Français (SYSAAF). Cette domestication s'appuie sur le transfert de la procédure de sélection sur la croissance PROSPER, élaborée par l'INRA, complétée de critères de sélection sur la qualité des carcasses (teneur en lipides des filets, rendement à l'éviscération) et d'une reproduction généalogique assistée par empreintes génétiques. L'amélioration des carcasses et de la chair est aussi réalisée par monosexage et triploïdisation. Depuis 2000, le transfert de la congélation du sperme est effectif. En 2003, entre $80 \%$ et $100 \%$ de la production française bénéficie de juvéniles provenant de programmes de sélection. l'environnement, d'amélioration du bien-être des animaux et de respect de principes éthiques (Komen et al 2002).

Après avoir illustré les handicaps et les atouts des poissons en matière de domestication, le contexte historique et les spécificités de la pisciculture française, cet article présente les actions entreprises par les pisciculteurs français pour adapter le potentiel génétique de leurs espèces aux attentes du marché, que ce soit en matière d'amélioration génétique ou de reproduction.

\section{Handicaps et atouts des poissons pour une domestication rationnelle}

Plusieurs éléments constituent des contraintes pour la domestication des poissons : - le nombre d'espèces pouvant faire l'objet d'effort de domestication est très élevé : plus de 150 de par le monde ;

- la plupart des espèces piscicoles sont des prédateurs carnivores dont le comportement peut être agressif, surtout en phase juvénile ;

- contrairement aux animaux terrestres, les poissons n'ont que peu d'histoire commune avec l'homme ;

- les poissons sont des espèces poïkilothermes dont les capacités d'adaptation thermique limitent géographiquement la diffusion des efforts de domestication.

Inversement, plusieurs éléments sont favorables à une domestication rapide et efficace : - la plupart des espèces sont encore proches du génotype « sauvage » et présentent une variabilité génétique importante ;

- les poissons sont des animaux de petite taille, ce qui permet de concentrer l'effort de 
sélection sur des lignées spécialisées composées d'un nombre réduit d'animaux

- les femelles présentent une fécondité élevée, permettant des pressions de sélection importantes et une diffusion rapide des progrès ;

- les poissons présentent une plasticité physiologique propice à l'application de techniques de la reproduction qui maximisent les combinaisons génétiques à chaque nouvelle génération ;

- enfin, chez les poissons, les manipulations du niveau de ploïdie sont assez aisées et donnent généralement des animaux viables à l'état di-, tri- ou tétraploïde, aptitude particulièrement intéressante pour fixer des gènes d'intérêt ou stériliser des individus.

\section{Contexte historique de la domestication des poissons}

La production piscicole moderne ne date que de quelques décennies. La première trace écrite en situe le développement il y a 2500 ans en Chine. L'interdiction de la consommation de viande au Moyen Age en Europe a stimulé le développement de la pisciculture en étang dont celle de la carpe commune (Cyprinus carpio). Son élevage extensif et les échanges limités entre régions de production ont permis la création de races géographiques et leur relative domestication (Hollebecq et Haffray 1994).

La première fécondation artificielle, réalisée en 1842 en France, a suscité le développement d'établissements de "piscifacture " (Coste 1856). Plusieurs millions d'oufs de salmonidés ont alors été introduits dans les rivières ou expédiés à travers le monde. Le transfert de ce principe à la morue (Gadus moruha) en 1866 a initié des programmes de repeuplement à partir de reproducteurs sauvages aux USA et en Norvège jusqu'en 1952 (Shelbourne 1964), mais sans domestication.

Parallèlement aux actions de déversement d'individus de génotype sauvage, le début de la domestication de la truite arc-en-ciel (Oncorhynchus mykiss) remonterait à sa première reproduction artificielle en 1874 en Californie (Hershberger 1992). Lintroduction de cette espèce en Europe au début du XX ${ }^{\text {ème }}$ siècle a accompagné l'émergence de la pisciculture européenne. Les aliments composés et la mise en place d'une politique de prévention sanitaire ont accéléré ce développement vers 1960. Le transfert de ces acquis au saumon atlantique Salmo salar en Norvège, puis aux poissons marins en France et en Angleterre (bar Dicentrachus labrax, daurade Sparus aurata et turbot Scophthalmus maximus) dans les années 1980-1985, associé à l'amélioration de la survie larvaire ont été déterminants dans la diversification de la production européenne. Des essais sont tentés en France métropolitaine sur le maigre (Argyrosomus regius) et dans les DOM-TOM sur le tilapia rouge, l'ombrine tropicale (Sciaenops ocellatus) et le cobia
(Rachycentron canadum), ou en Europe sur le pagre (Pagrus pagrus), les soles (Solea solea et $S$. senagalensis), le dentex (Dentex dentex), la sériole (Seriola dumerili), le pageot (Pagellus bogaraveo), la morue, le flétan (Hyppoglossus hyppoglossus) ou le thon rouge (Thynnus thynnus).

\section{La pisciculture française : limitée en volume mais diversifiée}

En 2002, la production piscicole française était de l'ordre de 60000 tonnes pour 210 millions d'euros de chiffre d'affaires. Cette petite filière compte environ 680 entreprises installées sur 870 sites et emploie directement plus de 2000 personnes, hors emplois induits (données CIPA, SCEES Agreste Primeur n ${ }^{\circ} 54$ et $59)$. Dominée en volume et en valeur par la truite arc-en-ciel, la filière s'est diversifiée, tant en termes d'espèces ${ }^{(1)}$, que de produits commercialisés (diversité des poids à l'abattage et des modes de transformation) : $10000 \mathrm{t}$ de grandes truites triploïdes stériles (3 à $4 \mathrm{~kg}$ ) pour le fumage ou la découpe, $10000 \mathrm{t}$ de truite à filet $(0,8$ à $1,2 \mathrm{~kg}), 27000 \mathrm{t}$ de truite de taille portion ( $300 \mathrm{~g}), 2500 \mathrm{t}$ de bar et de daurade (600 g à 1,2 kg), $2500 \mathrm{t}$ de bar et daurade de taille portion (300 g), $150 \mathrm{t}$ de caviar de truite et $10 \mathrm{t}$ de caviar d'esturgeon. Cette évolution répond à la demande du marché pour du poisson d'origine diversifiée et de plus grande taille. Elle répond aux mêmes logiques que celles des autres filières animales : segmentation du marché, report des gains sur les étapes permettant le maintien d'une plus-value, c'est-à-dire la découpe et la transformation, qui nécessitent des poissons de plus grande taille.

\section{Emergence du métier de sélectionneur}

Les premiers travaux sur l'évaluation des bases génétiques de la croissance, premier critère d'intérêt zootechnique, ont été conduits simultanément sur la truite arc-en-ciel et le saumon atlantique en Norvège (Gjedrem, 1975), en France (Chevassus 1976), en Allemagne et aux USA (Kincaid et al 1977).

Un programme expérimental de sélection sur la vitesse de croissance et la maturation tardive (sélection généalogique combinée) a été entrepris en Norvège dès 1976 chez le saumon atlantique (4 lignées) et la truite arc-enciel (3 lignées) avec 200 familles par génération. Les producteurs se sont regroupés en 1986 pour assurer la continuité de cet investissement en augmentant progressivement le nombre de caractères sélectionnés (teneur en lipides et pigmentation du filet, résistance à la furonculose et à la vibriose d'eau froide) et le nombre de familles (400 pour le saumon). Ce mode de sélection est

(1) Truite arc-en-ciel (44 $000 \mathrm{t}$ ), carpe commune (6000 t), bar (3500 t), daurade (1500 t), saumon atlantique (1200 t), turbot (1000 t), truite fario élevée en rivière (2000 $\mathrm{t}$ ) ou en mer $(500 \mathrm{t})$, ombrine tropicale $(450 \mathrm{t})$, omble de fontaine $(250 \mathrm{t})$, esturgeon sibérien Acipenser baeri (150 t), tilapia (200 t), silure glane Silurus glanis (200 t), maigre (100 t), omble alpin Salvelinus alpinus (50 à $100 \mathrm{t})$ 
basé sur l'élevage des familles séparément jusqu'à leur regroupement à partir d'une taille permettant un marquage individuel. Cinq programmes de sélection de ce type existent maintenant en Norvège.

Ce modèle a progressivement été appliqué en sélection commerciale au Canada sur le saumon atlantique (Friars et al 1990), aux USA sur le saumon coho (Hershberger et al 1990) et la truite arc-en-ciel (Parson 1990) et en Ecosse (Marine Harvest) sur le saumon atlantique. Aujourd'hui, plus d'une trentaine de programmes de ce type sont conduits sur le saumon atlantique (16) et la truite arc-enciel (6), mais aussi sur l'omble arctique (Suède, Islande, Canada), le tilapia du Nil (Philippines), le turbot (Espagne), la daurade (Italie, Grèce et Espagne), les crevettes tropicales (Asie et en Amérique de Sud), l'huître creuse Crassostrea gigas (USA et Tasmanie) et la carpe commune (Inde).

Un doublement du poids à âge égal est rapporté après 7 générations de sélection chez le saumon, doublement associé à des améliorations de l'indice de conversion alimentaire et du rendement à l'éviscération, à une stabilité de la teneur en lipides du filet (Thodesen $e t$ al 1999) et à une diminution de l'agressivité (Mork et al 1999). Il est difficile d'évaluer la part du progrès due à la sélection ou à la domestication car ces programmes n'entretiennent pas de lignée témoin. Dans un des rares cas pour lesquels une lignée témoin a été entretenue, Hershberger et al (1990) estiment qu'au minimum un tiers du progrès observé après 4 générations de sélection chez le saumon coho $(+60 \%)$ serait dû à la domestication. Si ce type de programme s'avère généralement efficace, il n'en demeure pas moins onéreux et peu adapté à une production de volume limité ou concernant des espèces dont la zootechnie est encore mal maîtrisée.

Parallèlement à cette démarche, l'INRA, dans le cadre de la station expérimentale mixte INRA-IFREMER (SEMII, Sizun - 29), a optimisé la sélection individuelle sur la croissance chez la truite fario Salmo trutta à partir de certaines spécificités des salmonidés : fécondité maternelle élevée, effets maternels (âge des mères, taille de l'œuf, date de ponte). Cette PROcédure de Sélection Par Epreuves Répétées, d'acronyme PROSPER, moins onéreuse que la sélection généalogique, a permis un gain de poids de $120 \%$ en 5 générations (héritabilité réalisée de 0,40 ; Vandeputte et al $2002 \mathrm{~b}$ ). Après 3 générations de sélection, il est observé, à poids égal à l'abattage, une baisse de la teneur en lipides du filet et du rendement au filetage (1\% et $1 \%$; Bonnet et al 1999), un développement plus important des myoseptes ventraux (Marty-Mahé et al 2004), sans altération de l'efficacité alimentaire (Sanchez et al 2001) ni du rendement à l'éviscération ou des caractéristiques sensorielles de la chair (Haffray et al 1999).

\section{La section aquacole du SYSAAF}

En 1990, simultanément à l'obtention des premières estimations de réponse à la sélec- tion expérimentale PROSPER, le Syndicat des Sélectionneurs Avicoles Français (SYSAF), regroupant les sélectionneurs d'espèces avicoles (Stevens 1991), a été chargé par le ministère de l'Agriculture et de la Pêche d'évaluer la faisabilité de la mise en œuvre de programmes d'amélioration génétique des salmonidés. Le 1er juin 1991, le SYSAF a étendu son activité aux salmonidés en devenant le Syndicat des Sélectionneurs Avicoles et Aquacoles Français (SYSAAF). Chaque entreprise est propriétaire de ses lignées et détermine ses objectifs commerciaux. Le SYSAAF intervient en tant que conseiller pour l'orientation des programmes de sélection, le traitement statistique des données, l'amélioration des procédures et du statut hygiénique et sanitaire des reproducteurs. Cette approche mutualise le coût de l'expertise correspondant à des compétences techniques sensibles et coûteuses, mais aussi susceptibles d'évoluer rapidement sous l'effet de la concurrence ou de l'innovation.

En 2003, le SYSAAF compte 14 entreprises qui sélectionnent 7 espèces aquacoles (truite arc-en-ciel, truite fario, omble de fontaine, bar, turbot, daurade, huître creuse). Entre $80 \%$ et $100 \%$ de la production de ces espèces bénéficie de juvéniles sélectionnés. Plusieurs points peuvent être observés :

- trois phases se sont succédé avec l'arrivée des entreprises productrices de salmonidés (1991-1992), puis celles de poissons marins (1993-1996) et récemment les écloseries d'huître creuse (2001) ;

- sur les 24 entreprises ayant adhéré au SYSAAF entre 1991 et 2003, seules 14 conduisent encore un travail d'amélioration génétique en 2003 ;

- des cessations d'activité, des rachats ou des restructurations se sont opérés, le plus souvent avec rachat des lignées et poursuite du travail initié

- seules les entreprises présentant un chiffre d'affaires minimum d'environ 3 millions d'euros ont pu investir sur la durée ;

- cette spécialisation et ces investissements se sont accompagnés du développement d'une activité d'exportation d'œufs ou de juvéniles vers l'Europe, le pourtour méditerranéen, le Golfe Persique et la Chine, et par la création de premières unités de multiplication à l'étranger ;

- les entreprises, avec leurs filiales européennes, produisent de l'ordre de $60 \%$ des œufs européens de truite arc-en-ciel (550 millions d'oeufs), $80 \%$ des juvéniles de turbot (7 millions), 8 à $10 \%$ des alevins de bar (25 millions), de daurade (20 millions), $70 \%$ des naissains d'écloserie d'huître creuse et environ 50 à $70 \%$ de la production nationale de truite arc-en-ciel et de poissons marins.

\section{Amélioration de la croissan- ce par la sélection}

La taille limitée de la filière française, la diversité des espèces, la concurrence entre les entreprises et la lourdeur des programmes de sélection de type généalogique ont conduit les pisciculteurs à investir dans des programmes de sélection individuelle. Dix-sept 
lignées de 4 espèces (truite arc-en-ciel, truite commune ou fario élevée en eau douce, turbot et daurade) sont sélectionnées (figure 1) selon des schémas de sélection de type PROSPER ou de type " massale » plus classique (bar et omble de fontaine). Ces programmes bénéficient en outre d'améliorations (figure 2) concernant la reproduction, la gestion génétique ou la mâ̂trise de la qualité (voir plus loin).

Conformément aux recommandations internationales, les effectifs génétiques efficaces minimaux de reproducteurs sont supérieurs à 100 pour chacune des lignées. Les intervalles entre générations sont de 2 ans pour les salmonidés et de 3 à 5 ans pour les poissons marins. Les pressions de sélection sont de l'ordre de 5 à $1 \%$. De 150000 à 200000 mesures sont réalisées par génération et par lignée. Ont été obtenues 4 générations de sélection chez la truite arc-en-ciel, 2 chez le turbot et 1 chez la daurade, ces dernières espèces et le bar disposant déjà de 1 à 3 générations de domestication avant la mise en

Figure 1. Evolution du nombre de lignées de poissons sélectionnées par les entreprises adhérentes du SYSAAF de 1991 à 2003.

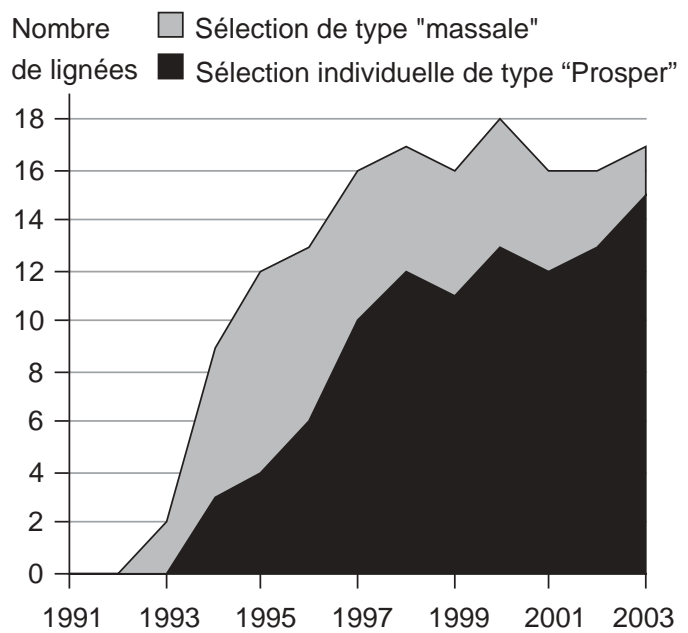

œuvre des programmes de sélection.

Chez les poissons marins, la difficulté à individualiser les pontes a conduit à limiter les effectifs de mères et à gérer la sélection sous forme de groupes de reproduction, un flux de gènes entre ces groupes de reproduction étant réalisé par croisements rotatifs avec du sperme frais ou congelé.

D'autres aptitudes devraient être co-sélectionnés, telles que l'aptitude à subir des manipulations lors des tris ou de la reproduction. Les élevages trutticoles diffèrent par de nombreux facteurs : la conduite zootechnique, le pH de l'eau (de 5 dans les Landes à 7,5 dans les Pyrénées), la température constante $\left(11^{\circ} \mathrm{C}\right.$ ou $\left.14^{\circ} \mathrm{C}\right)$, moyennement variable $\left(8\right.$ à $\left.16^{\circ} \mathrm{C}\right)$ à extrêmement variable $\left(2,5\right.$ à $\left.22,5^{\circ} \mathrm{C}\right)$, la turbidité, le profil d'oxygène dissous ou les densités d'élevage. Il est probable que des aptitudes différentes s'établiront progressivement entre lignées pouvant conduire à d'éventuelles interactions génotype $\mathrm{x}$ milieu.

Le coût de la sélection-domestication est estimé de l'ordre de 16,6 euros/t à l'échelle de la filière, à raison de 7,8 euros/t chez les salmonidés et 90 euros/t chez les poissons marins. Le coût pour les poissons marins s'explique par la part limitée du grossissement réalisée en France et par la production de juvéniles destinés à l'exportation (entre 40 et $50 \%$ du chiffre d'affaire). Plus globalement, cet investissement représente de l'ordre de 0,4 $\%$ du chiffre d'affaires de la filière française.

Des programmes de sélection généalogique multicaractères et multigénération permettraient une sélection sur apparentés sur des critères létaux (rendements de découpe, pigmentation de la chair, résistance à divers pathogènes) et l'utilisation de méthodes d'évaluation génétique plus classiques telles que le BLUP (Best Linear Unbiased Predictor). Cependant, l'élevage de familles séparément jusqu'au marquage est associé à des effets d'environnement commun importants. Une amélioration du rapport

Figure 2. Exemple de schéma de sélection conduit chez la truite arc-en-ciel sur la base de la procédure de sélection individuelle de type PROSPER complétée des innovations sur la prise en compte de critères de qualité et sur l'intégration de la reproduction généalogique par assignation de parenté par empreintes génétiques.

3. Indexation qualitative de 2500 candidats à 16 mois, sélection sur index et identification de 500 candidats Pression de sélection de $20 \%$

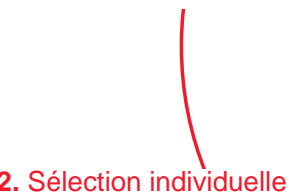

sur la longueur et le poids par tris successifs de remise en compétition entre 4 et 16 mois

Pression de sélection de $3 \%$
Schéma de sélection

4. Génotypage des 500 candidats

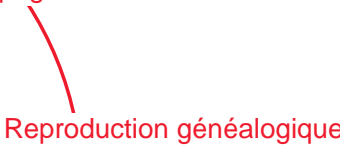

des candidats

$$
50 \text { mères * } 50 \text { pères }
$$
création de 1000 familles
Multiplication instantanée

ou spécialisée (élites) 
qualité/prix de tels programmes est attendue par le mélange des familles dès l'éclosion et l'assignation de parenté a posteriori par empreintes génétiques. Ce principe a été testé avec succès sur 184 familles de truite arc-enciel (Chevassus et al 2002) et 30 familles de bar (Saillant et al 2002) et pourrait constituer la prochaine étape de l'évolution des programmes de sélection.

\section{Maîtrise de la reproduction}

La maîtrise de la reproduction constitue un facteur déterminant de la conduite efficace des programmes de domestication et d'amélioration génétique.

La disponibilité en ovocytes la semaine ou le jour souhaité réduit les effets environnementaux de date de ponte, tout en limitant les effectifs en sélection en s'affranchissant de l'étalement de la période d'ovulation. Après des essais de divers produits commerciaux sous contrôle de l'Agence française du Médicament (Ovaprim ${ }^{\mathrm{TM}}$, Reproboost ${ }^{\mathrm{TM}}$ ), les sélectionneurs, sur prescription vétérinaire, induisent hormonalement la ponte d'environ 500 femelles/an avec du Décapeptyl ${ }^{\mathrm{TM}}$ (Breton et al 1990), préparation à usage médical à base de triptoréline, hormone gonadolibérine (GnRH) de synthèse présentée sous forme retard. Un effort important a été réalisé pour maîtriser la technique d'induction, avec en particulier la participation à une étude concernant l'obtention, par la société Intervet, d'une autorisation européenne de mise sur le marché (AMM EU/2/03/040/001) pour le Gonazon ${ }^{\mathrm{TM}}$. Cette étude a porté sur plus de 1760 femelles de truite arc-en-ciel, de saumon atlantique et d'omble arctique (Haffray et al 2004b).

La qualité de la laitance constitue un deuxième point-clé. Des mesures de motilité des spermes des extraits testiculaires des néomâles (femelles XX masculinisées, voir plus loin) ont montré une grande variabilité de ce caractère en pisciculture. Après différents travaux, le dilueur de collecte et de maturation des spermatozoïdes Storfish ${ }^{\mathrm{TM}}$ a été développé (accord de Licence INRA-IMVSYSAAF). Ce dilueur permet d'atteindre 80 à $90 \%$ de motilité après 3 heures d'incubation à $4^{\circ} \mathrm{C}$ et de conserver cette motilité sur au minimum 5 jours chez les salmonidés, la daurade, le turbot, la carpe et le silure glane (Maisse $e t$ al 1998). Cette innovation réduit le nombre de mâles nécessaire en multiplication et améliore l'hygiène des laitances par adjonction d'antibiotiques. L'intérêt de l'emploi des iodophores lors de la fécondation et du gonflement des oufs a été confirmé pour diminuer le risque de transmission verticale de la nécrose pancréatique infectieuse, sans l'empêcher (Dorson et al 1996 et 1997).

\section{Amélioration de la qualité par le monosexage}

Dès la création de la section aquacole du SYSAAF, la maîtrise génétique de la qualité est apparue prioritaire. Différentes actions ont été conduites : monosexage génétique des espèces pour lesquelles cette technique pouvait être intéressante, optimisation de la stérilisation et évaluation de ses effets, et enfin mise au point de critères de sélection.

Le poids à l'abattage est généralement atteint après la maturation sexuelle des mâles, plus précoces d'un an que les femelles. La maturation réduit la teneur en lipides et en pigments des filets de salmonidés, altère l'indice de conversion alimentaire, fragilise les reproducteurs et favorise l'implantation d'agents pathogènes pouvant entraîner leur mort (saprolégniose de salmonidés, par exemple). Diverses solutions permettent de limiter l'impact négatif de la reproduction telles que l'application de régimes photopériodiques décalant de quelques mois ce phénomène (Maisse et Breton 1996) ou la sélection de lignées à maturation tardive. Cette dernière alternative est intéressante dans un contexte de production d'animaux à croissance lente, mais la corrélation génétique négative avec la croissance limite son intérêt dans un contexte d'amélioration de la vitesse de croissance.

L'INRA a développé et transféré la possibilité de produire des populations $100 \%$ femelles en combinant l'élimination du gène mâle (déterminisme homogamétique XX femelle et XY mâle) et l'inversion hormonale des reproducteurs femelle XX au stade alevin par voie orale (Breton et al 1996). Alors même que les femelles croissent $10 \%$ moins vite que les mâles, cette innovation intéresse aujourd'hui 80 à $90 \%$ de la filière, l'amélioration de la qualité par l'élevage de poissons non immatures constituant une priorité plus importante que celle de la croissance.

En l'absence de réglementation sur l'utilisation des traitements d'inversion sexuelle, une première action a consisté en l'élaboration d'une réglementation nationale qui a ensuite été reprise au plan européen (Directive 96/22/CE du 29/04/1996). Les élevages pratiquant cette inversion le font sous le contrôle de leur Direction des Services Vétérinaires ; les reproducteurs traités sont marqués de façon visible et pérenne et leurs carcasses envoyées à l'équarrissage, ils ne peuvent être proposés à la consommation humaine.

Des travaux de recherche ont ensuite été entrepris chez plusieurs espèces pour évaluer l'importance du dimorphisme sexuel et tenter d'obtenir des populations monosexe :

- chez le turbot, qui présente un dimorphisme de croissance à l'avantage des femelles $(+10$ à $20 \%$ à partir de 800 g), des travaux ont conclu à un déterminisme génétique de type ZZ/ZW limitant la rapidité d'obtention de monosexes femelles ;

- chez le bar, qui présente aussi un dimorphisme de croissance à l'avantage des femelles de 10 à $20 \%$ (Saillant et al 2001) et 80 à $100 \%$ de mâles en élevage, il a été démontré un déterminisme génétique du sexe lié à la température (Saillant et al 2002) ;

- chez l'omble de fontaine, il a été démontré la nécessité de compléter les traitements d'inversion sexuelle par des balnéations dès la semaine précédant l'éclosion ; 
- chez le silure, qui présente un dimorphisme à l'avantage des femelles (Haffray et al 1998).

\section{Amélioration de la qualité par la stérilisation}

Le monosexage femelle ne résout pas les problèmes liés à la maturation des femelles. Chez les espèces inférieures comme les poissons, mais aussi les reptiles, les amphibiens, les mollusques et les crustacés, il est possible de produire des individus triploïdes avec 2 lots de chromosomes de leur mère et une de leur père en inhibant la répulsion du second globule polaire, en plongeant les oeufs dans de l'eau à $26,5^{\circ} \mathrm{C}$ pendant 20 minutes, 20 minutes après la fécondation, par exemple chez la truite arc-en-ciel (Breton et al 1996). La production de truites stériles, à l'image des castrations pratiquées en production bovine, porcine ou avicole, permet de commercialiser des produits immatures, même si ce génotype présente une croissance inférieure de l'ordre de $10 \%$ à celle du diploïde femelle et de $20 \%$ à cellle du diploïde mâle. Les polyploïdes ne constituent pas des OGM (Directive 90/220/CEE) puisque ce type de génotype est rencontré dans la nature et que la majorité des végétaux que nous consommons (pommes, agrumes, pomme de terre, blé dure, kiwi, blé tendre, ail, fraise) sont polyploïdes, l'AFSSA ayant rendu un avis concluant sur la possibilité de consommer sans risque les huîtres triploïdes (03/11/2001). Cette technique est aussi recommandée par diverses organisations non gouvernementales pour limiter les impacts génétiques sur les populations sauvages des échappés d'élevages (Conseil International pour l'Exploitation de la Mer, North Atlantic Salmon Conservation Organisation, FAOFood and Agriculture Organization of the United Nations, etc).

La triploïdisation par traitement thermique des oufs était disponible dès le milieu des années 1980 (Chevassus et al 1985). Après la validation d'une méthode rapide d'évaluation du taux de triploïdie par cytométrie en flux sur alevin empruntée aux végétaux (Lecommandeur et al 1994), différentes expé-

Figure 3. Evolution du nombre cumulé total d'alevins et d'œufs embryonnés analysés par cytométrie en flux par le SYSAAF entre 1992 et 2003.

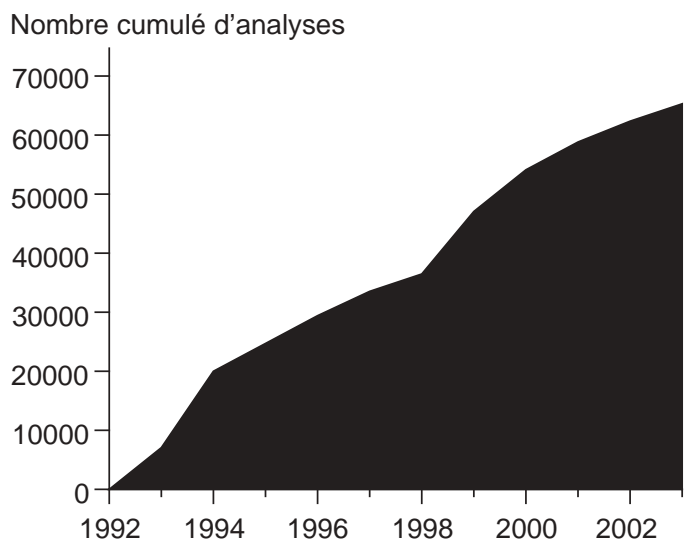

rimentations ont confirmé l'intérêt d'une méthode alternative par traitement hyperbare. Divers travaux ont montré l'absence d'interaction niveau génétique $\mathrm{x}$ ploïdie et donc le transfert du progrès génétique acquis en phase diploïde à la phase triploïde (Choubert et al 1997, Bonnet et al 1999). De l'ordre de 10 000 t de truites triploïdes (3 à 4 kg) sont produites, essentiellement pour le fumage, grâce au développement d'enceintes de traitement hyperbare de grands volumes d'œufs (10 l) et d'un service d'analyse de ploïdie par cytométrie en flux (figure 3).

L'optimisation de la triploïdisation et l'évaluation de la performance des triploïdes a été initiée chez d'autres espèces : silure glane (Linhart et al 2001), omble arctique (Gillet et al 2001), bar (Peruzzi et al 2004), turbot, daurade (Haffray et al 2004a) et omble de fontaine. L'intérêt qualitatif cette technique semble cependant limité chez les poissons marins : ces derniers ayant une chair blanche, ils ne peuvent présenter de baisse de pigmentation lors de la reproduction, au contraire des salmonidés à chair rose.

\section{Sélection sur des critères de qualité}

Compte tenu de la méthode de sélection individuelle pratiquée, la principale difficulté réside dans le développement de méthodes indirectes de prédiction des caractères qui soient non létales, précises, peu onéreuses et rapides à mettre en oeuvre au bord des bassins.

Un premier travail a porté sur la faisabilité d'une évaluation de la teneur en lipides des filets par micro-ondes (2 GHz) avec le Torry Fish Fat Meter. Différentes mesures donnent un coefficient de corrélation de l'ordre de 0,7 avec les méthodes d'estimation de la teneur en lipides par mesure chimique ou par IRM chez la truite arc-en-ciel et le bar (Douirin et al 1998, Haffray et al 2000).

Un deuxième travail a porté sur la validation d'un prédicteur du rendement à l'éviscération $\left(\mathrm{r}^{2}=0,5\right)$ : l'épaisseur de la paroi musculaire abdominale, estimée par ultrasons $(7,5 \mathrm{MHz})$ chez la truite arc-en-ciel. Ce critère est complété par un descripteur anatomique (note de 0 à 4 ) de la répartition des principales masses corporelles (tête, abdomen, format, dos).

Le déterminisme génétique de ces prédicteurs a été évalué sur 16 demi-fratries diploïdes et triploïdes de truites arc-en-ciel et fario (Haffray et al 1999) et sur 182 familles mélangées de truite arc-en-ciel (Chevassus et al 2002), par mesure de l'héritabilité réalisée suivant 2 générations de sélection divergentes (héritabilité réalisée de 0,33 pour l'épaisseur abdominale et 0,25 pour la mesure en Fat Meter ; Quillet et al 2002). Ces deux caractères sont introduits dans les programmes de sélection de la truite depuis 1995 et transférés au bar, à la daurade et au turbot. En fonction des pressions de sélection, des gains de 0,3 à 0,5\% par génération sont atten- 
dus sur le rendement à l'éviscération ou sur la teneur en lipides des filets

Parallèlement à ces travaux, différentes méthodes de caractérisation de la qualité de la chair ont été développées pour valider les caractères non létaux : teneur en lipides des filets par RMN sans solvants (Toussaint et al 2002), répartition de la teneur en lipides, de la pigmentation et des différents tissus d'une darne par IRM (Collewet et al 2001) ou par vision numérique (Marty-Mahé et al 2004).

\section{Gestion des ressources génétiques}

Limpossibilité d'un marquage individuel précoce ne permet pas d'avoir accès à la généalogie des individus. Des pertes de variabilité génétique non maîtrisées sont donc probables et l'on sait qu'une perte de variabilité (= augmentation de consanguinité) de $10 \%$ induit une perte de performance de l'ordre de $5 \%$ chez la truite par exemple (Kincaid 1976).

Lintérêt des plans de fécondation factorielle, préconisés dans le cadre de PROSPER pour maximiser la conservation de la variabilité génétique, a été confirmé par simulation (Dupont-Nivet et al 2004).

La connaissance de la généalogie a été à la base de la mise en œuvre des programmes de sélection chez les animaux domestiques. Cette impossibilité initiale dans le cadre des programmes de sélection individuelle de type PROSPER a été contournée grâce à l'usage des empreintes génétiques. Des marqueurs microsatellites ont été d'abord développés chez le turbot et la truite arc-en-ciel (Estoup et al 1998) puis la daurade royale (Launey et al 2003). Une évaluation de leur intérêt a été réalisée chez la truite arc-en-ciel lors d'une génération de sélection PROSPER (Haffray et al 2001). Ce travail a conclu à une perte limitée de variabilité génétique $(0,4 \%$ de la variabilité initiale), avec environ $90 \%$ des parents représentés dans la descendance sélectionnée, mais avec une forte variabilité du

Figure 4. Nombre annuel de poissons génotypés par empreintes génétiques par les sélectionneurs de 1997 à 2003.

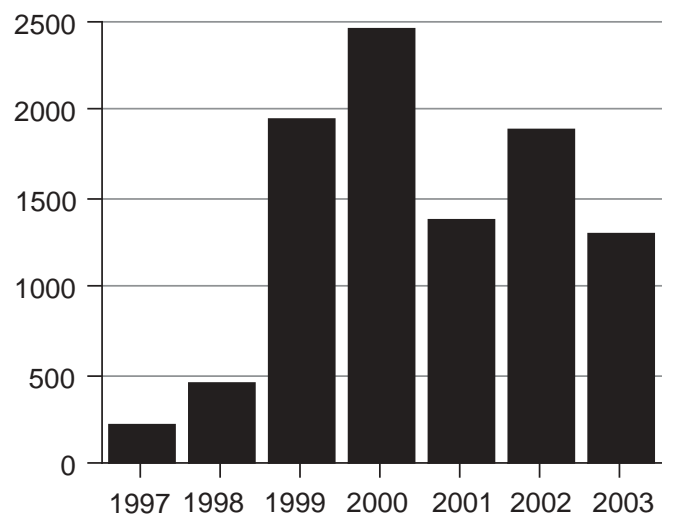

nombre de descendants par parent (d'un facteur 1 à 10).

Un travail de simulation a montré qu'un nombre minimum de 8 à 10 marqueurs était nécessaire pour obtenir des assignation de parenté de l'ordre de $95 \%$ chez la truite et le turbot (Estoup et al 1998). Les analyses ont été automatisées par LABOGENA pour la truite arc-en-ciel, le bar, la daurade et le turbot. Cette technologie est utilisée par les sélectionneurs pour maîtriser l'évolution de la consanguinité des lignées et optimiser les plan de fécondation sur proposition du SYSAAF et pour initier une multiplication s'appuyant sur des animaux élites. Près de 10 000 poissons ont été génotypés dans cet objectif depuis 1997 (figure 4).

Un travail préparatoire à la gestion génétique de la carpe en France a été initié. Il conclut à une variabilité génétique limitée des populations des Dombes, du Forez, de l'Alsace et de Sologne et à l'absence de différence de morphologie et de performances de croissance et de rendement de découpe entre ces populations (Vandeputte et al 2002a).

\section{La cryoconservation pour la conservation des ressources génétiques}

La congélation du sperme s'est avérée déterminante chez de nombreuses espèces dans l'organisation des programmes d'amélioration génétique pour diffuser ou conserver le potentiel génétique ou pour évaluer le progrès génétique. Dès 1994, des travaux ont permis le développement d'un dilueur de congélation du sperme multispécifique et un dilueur de lavage des ovocytes : Cryofish ${ }^{\mathrm{TM}}$ et Ovafish $^{\mathrm{TM}}$ (accord de Licence INRA-IMVSYSAAF), permettant l'obtention de 60 à $70 \%$ d'éclosion chez la truite arc-en-ciel (Maisse $e t$ al 1998). Cette technologie est utilisée en routine avec congélation sur site depuis 2000 (figure 5).

Figure 5. Nombre annuel de pères et de paillettes congelées (0,5 ml ; IMV Technologies) par les sélectionneurs de poissons de 2000 à 2003.

Nombre de mâles

Nombre de paillettes

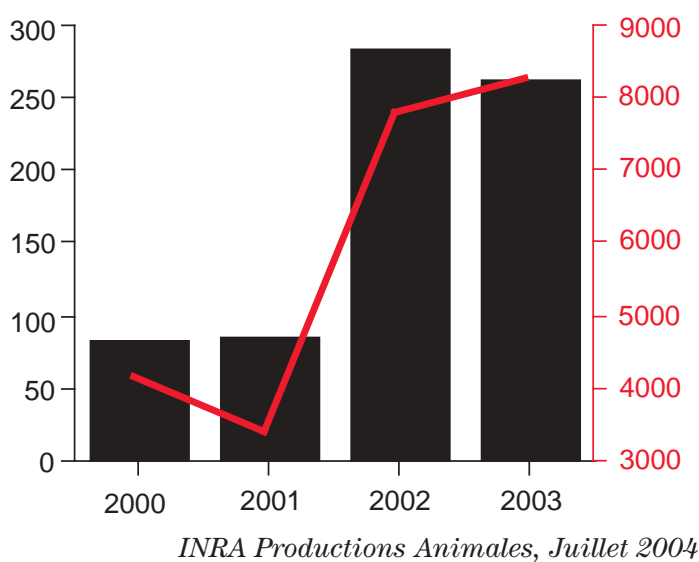




\section{Conclusions et perspectives}

En se regroupant dans le cadre du SYSAAF, les éleveurs ont développé une capacité d'expertise collective. Lintégration de différentes technologies permet la conduite de programmes de sélection sur la croissance et la qualité des carcasses tout en intégrant des préoccupations de gestion et de conservation des ressources génétiques.

L'évolution et la segmentation des marchés, les barrières sanitaires et la concurrence étrangère (sélection généalogique) pourraient, de même, conditionner la nature des investissements des sélectionneurs ainsi que la diffusion du progrès génétique. L’utilisation de nouvelles technologies, telles que l'assignation de parenté par marqueurs génétiques, l'identification de QTL de résistance à des pathogènes ou d'aptitudes qualitatives particulières et la transgenèse- pourrait, de même, radicalement influer sur les orientations de programmes de sélection et sur l'importance de la concurrence.

Plus globalement, si le potentiel génétique commence à faire partie des termes de l'échange commercial, l'application de programmes rationnels de domestication chez les espèces nouvelles (maigre, ombrine, omble de fontaine et omble alpin, silure) ou dont le volume de production est limité en France (carpe commune, tilapia), chez les mollusques (huître creuse, palourde, ormeau, huître plate, escargots) ou chez les crustacés (crevettes tropicales) constitue un sujet d'intérêt pour nombre d'acteurs de ces filières. L'aptitude des professionnels à s'organiser pour réduire le coût de mise en œuvre de ces programmes devrait conditionner le succès de la domestication de ces espèces.

\section{Remerciements}

L'équipe du SYSAAF tient à remercier les cadres techniques et les chefs des entreprises de sélection, les personnels techniques et administratifs du ministère de l'Agriculture et en particulier : Bernard Chevassus, André Fauré, Jean-Marie Blanc, Gérard Maisse, Alex Fostier, Benoit Fauconneau, Bernard Breton, René Guyomard, Edwige Quillet, Marc Vandeputte, Francine Krieg, Michel Dorson, Mathilde Dupont-Nivet, Françoise Médale, Sophie Launey, Arnaud Estoup, Karim Garbi et Joël Aubin de l'INRA, Béatrice Chatain de l'IFREMER, Marie-France Cazalère, Odile Dupuis et Isabelle Terrier du ministère de l'Agriculture et de l'OFIMER, Maryvonne Boscher, Bertrand Bedhom et Yves Amigues du laboratoire LABOGENA, les anciens collaborateurs du SYSAAF Paul Stevens, Michel Reffay, Eric Guinebert, Cécile Vauchez, Cyrilles Deshayes et Stéphane Bonnet, et les deux relecteurs qui ont amélioré le texte de cet article.

\section{Références}

Bonnet S., Haffray P., Blanc J.M., Vallée F., Vauchez C., Faure A., Fauconneau B., 1999. Genetic variation in growth parameters until commercial size in diploid and triploid freshwater rainbow trout (Oncorhynchus mykiss) and seawater brown trout (Salmo trutta). Aquaculture, 173, 359-375.

Breton B., Weil C., Sambroni E., Zohar Y, 1990. Effects of acute versus sustained administration of $\mathrm{GnRHa}$ on $\mathrm{GtH}$ release and ovulation in the rainbow trout, Oncorhynchus mykiss. Aquaculture, 91, 373-383.

Breton B., Quillet E., Jalabert B., 1996. Contrôle de la reproduction et du sexe chez les poissons d'élevage. INRA Prod. Anim., numéro hors série « 50 ans de recherches en productions animales », 17-26.

Chevassus B., 1976. Variabilité et héritabilité des performances de croissance chez la truite arc-en-ciel (Salmo gairdnerii richardson). Ann. Génét. Sél., anim., 8, 273-283.

Chevassus B., Quillet E., Chourrout D., 1985. La production de truites stériles par voie génétique. Piscic. Fr., 78, 10-19.

Chevassus B., Dupont-Nivet M., Mauger S., Haffray P., Vandeputte M., 2002. Estimation of heritabilities in two groups of rainbow trout (Oncorhynkus mykiss) with families mixed together since eyed stage. 7th World Congress on Genetics Applied to Livestock Production, Montpellier, August 19-23, 2002. Communication n06-09 (abstract), 147.

Choubert G., Blanc J.M., Vallée F., 1997. Colour measurement, using the CIELCH colour space of muscle of rainbow trout, Onchorhynchus mykiss (Walbaum), fed astaxanthin: effect of family, ploidy, sex, and location of reading. Aquac. Res., 28, 15-22.

Collewet G., Davenel A., Toussaint C., Akoka S., Médale F., Fauconneau B., Haffray P., 2001. Magnetic Resonance Imaging as a tool to quantify the adiposity distribution in fish. In: Webb G.A., Belton P.S., Gil A.M., Delgadillo I. (eds), Magnetic resonance in food science: a view to the future,
252-258. The Royal Society of Chemistry, Cambridge (UK).

Coste M., 1856. Instructions pratiques sur la pisciculture. Librairie Victor Masson, Paris.

Dorson M., Torhy C., Billard R., Saudrais C., Maisse G. Haffray P., Hollebecq M.G., 1996. Nécrose pancréatique infectieuse des Salmonidés : évaluation de méthodes destinées à couper la transmission par l'oeuf. Bull. Fr. Pêche Piscic., 340, 1-14.

Dorson M., Rault P., Haffray P., Torhy C., 1997. Water-hardening rainbow trout eggs in the presence of iodophor fails to prevent the experimental egg transmission of infectious pancreatic necrosis virus. Bull. Eur. Ass. Fish Pathol., 17, 13-16.

Douirin C., Haffray P., Vallet J.L., Fauconneau B., 1998 Détermination de la teneur en lipides des filets de truite arc-en-ciel Oncorhynchus mykiss par le Torry Fat Meter ${ }^{\circledR}$ Sci. Alim., 18, 527-535.

Dupont-Nivet M., Vandeputte M., Haffray P., Chevassus B., 2004. Factorial and partly factorial mating designs are efficient designs for conservation of genetic variability during selection. Livest. Prod. Sci. (soumis).

Estoup A., Gharbi K., SanCristobal M., Chevalet C., Haffray P., Guyomard R., 1998. Parentage assignment using microsatellites in turbot (Scophtalmus maximus) and rainbow trout (Oncorhynchus mykiss) hatchery populations. Can. J. Fish. Aquat. Sci., 55, 715-725.

Friars G.W., Bailey J.K., Coombs K.A., 1990. Correlated responses to selection for grilse length in Atlantic salmon. Aquaculture, 85, 171-176.

Gillet C., Vauchez C., Haffray P., 2001. Triploidy induced by pressure shock in Arctic charr (Salvelinus alpinus): growth, survival and maturation until third year. Aquat. Living Resour., 14, 327-334. 
Gjedrem T., 1975. Possibilities for genetic gain in salmonids. Aquaculture, 6, 23-29.

Haffray P., Vauchez C., Vandeputte M., Linhart O., 1998. Different growth and processing traits in males and females of European catfish, Silurus glanis. Aquat. Living Resour., 11, 341-345.

Haffray P., Bonnet S., Blanc J.M., Laroche M., Vallet J.L., Weigel C., Chevassus B., Fauconneau B., 1999. Amélioration génétique et contrôle de la qualité en aquaculture. Compte rendu de fin de recherche. Programme Agriculture Demain 94 G 0200

Haffray P., Davenel A., Marty-Mahé P., Collewet G., Loisel P., Toussaint C., Médale F., Fauconneau B., 2000. Description des hétérogénéités de couleur de la chair de poisson. Compte rendu de fin de recherche. Programme AQS 1999 n98.P.0486.

Haffray P., Dupont-Nivet M., Amigues Y., Vandeputte M. Pincent C., Deshayes C., Chevassus B., 2001. Amélioration de la gestion des reproducteurs en trutticulture : Validation de l'intérêt des empreintes génétiques. Compte rendu final de la convention 033/00/C-OFIMER/CIPA.

Haffray P., Bruant J.S., Facqueur J.M., Fostier A., 2004a. Effect of triploidy on the zootechnical performances and gonad development in the gilthead seabream Sparus aurata (L.), a protandrous hermaphrodite Sparidae. Aquaculture (à paraître).

Haffray P., Enright W.J., Driancourt M.A., Mikolajczyk T., Rault P., Breton B., 2004b. Optimisation of breeding of Salmonids: Gonazon ${ }^{\circledR}$, the first officially approved inducer of ovulation in the EU. World Aquaculture Magazine (à paraître).

Hershberger W.K., 1992. Genetic variability in rainbow trout populations. Aquaculture, 100, 51-72.

Hershberger W.K., Myers J.M., Iwamoto R.N., McAuley W.C., Saxton A.M., 1990. Genetic changes in growth of coho salmon (Onchorhynchus kisutch) in marine net-pens, produced by ten years of selection. Aquaculture, 85, 187-198.

Hollebecq M.G., Haffray P., 1994. Revue bibliographique - L'amélioration génétique de la carpe commune Cyprinus carpio L. : état des connaissances. Bull. Fr. Pêche Piscic., 333, 93-124.

Kincaid H.L., 1976. Inbreeding in rainbow trout (Salmo gardneri). J. Fish. Res. Board Can., 33, 2420-2426.

Kincaid H.L, Bridges W.R, von Limbach B., 1977. Three generations of selection for growth rate in fall-spawning rainbow trout. Trans. Am. Fish. Soc., 106, 621-628.

Komen H., Haffray P., Kaushik S., New M., Olesen I., Liinamo A.E., 2002. Defining breeding goals for the future sustainable aquaculture. Aquaculture Europe, December 2002, 11-14

Launey S., Krieg F., Haffray P., Bruant J.S., Vannier A., Guyomard R., 2003. Twelve new microsatellite markers for gilthead seabream (Sparus aurata L.): characterisation, polymorphism and linkage. Mol. Ecol. Notes, 3, 457

Lecommandeur D., Haffray P., Philippe L., 1994. Rapid flow cytometry method for ploidy determination in Salmonid eggs. Fishery Management, 25, 345-350.

Linhart O., Haffray P., Ozouf-Costaz C., Flaj_han M., Vandeputte M., 2001. Comparaison of methods for hatchery-scale triploidization of European catfish (Silurus glanis). J. Appl. Ichthyol., 17, 247-255.

Maisse G., Breton B., 1996. Contrôle photopériodique de la saison de reproduction chez les salmonidés INRA Prod. Anim., 9, 71-77.
Maisse G., Labbé C., Ogier de Baulny B., Leveroni Calvi S., Haffray P., 1998. Cryoconservation du sperme et des embryons de poissons. INRA Prod. Anim., 11, 57-65.

Marty-Mahé P., Loisel P., Fauconneau B., Haffray P., Brossard D., Davenel A., 2004. Quality traits of brown trout Salmo trutta cutlets described by automated color image analysis. Aquaculture, 232, 225-240.

Mork O.I., Bjerkeng B., Rye M., 1999. Aggressive interac tion in pure and mixed groups of juvenile farmed and hatchery-reared wild Atlantic salmon Salmo salar L., in relation to tank substrate. Aquac. Res., 30, 571-578.

Parson E.J., 1990. Selective breeding programs in salmonids: a U.S. experience. Atelier de travail sur la génétique des salmonidés d'élevage au Québec. CAPQ, 1990, 21-31.

Peruzzi S., Chatain B., Saillant E., Haffray P., Menu B. Falguiere J.C., 2004. Production of meiotic gynogenetic and triploid sea bass, Dicentrarchus labrax L.: 1. Performances, maturation and carcass quality. Aquaculture, 230, 41-64.

Quillet E., Aubin J., Le Guillou F., Médale F., Fauconneau B., 2002. Response to mass selection for two traits related to fattening in rainbow trout (Oncorhynchus mykiss). 7th World Congress on Genetics Applied to Livestocks Production. Montpellier, August 19-23, 2002. Communication n ${ }^{\circ}$ 66-12 (abstract), p 94.

Shelbourne J.E., 1964. The artificial propagation of marine fish. Adv. Mar. Biol., 2, 1-83.

Saillant E., Fostier A., Menu B., Haffray P., Chatain B., 2001. Sexual growth dimorphism in sea bass (Dicentrarchus labrax). Aquaculture, 202, 371-387.

Saillant E., Fostier A., Haffray P., Menu B., Thimonier J. Chatain B., 2002. Temperature effects and genotype-temperature interactions on sex determination in the European sea bass (Dicentrarchus labrax L.). J. Exp. Zool., 292, 494-505.

Sanchez M.P., Chevassus B., Labbé L., Quillet E., Mambrini M., 2001. Selection for growth of brown trout (Salmo trutta) affects feed intake but not feed efficiency. Aquat. Living Resour., 14, 41-48.

Stevens P., 1991. La sélection avicole française et le rôle du SYSAAF. Aquarevue, 34, 23-26.

Thodesen J., Grisdale-Helland B., Helland S., Gjerde B. 1999. Feed intake, growth and feed utilisation of offspring from wild and selected Atlantic salmon (Salmo salar). Aquaculture, 180, 237-246.

Toussaint C., Médale F., Davenel A., Fauconneau B. Haffray P., Akoka S., 2002. Determination of the lipid content in fish muscle by a self-calibrated NMR relaxometry method: comparison with classical chemical extraction methods. J. Sci. Food Agric., 82, 173-178.

Vandeputte M., Prunet P., 2002. Génétique et adaptation chez les poissons : domestication, résistance au stress et adaptation aux conditions du milieu. INRA Prod. Anim., 15, 365-371.

Vandeputte M., Peignon E., Vallod D., Haffray P., Komen J., Chevassus B., 2002a. Comparison of growth performances of three French strains of common carp (Cyprinus carpio) using hem-isogenic scaly carp as internal control. Aquaculture, 205, 19-36.

Vandeputte M., Quillet E., Krieg F., Hollebecq M.G. Fauré A., Labbé L., Hiseux J.P., Chevassus B., 2002b. The "PROSPER" methodology on brown trout (Salmo trutta fario): four generations of improved individual selection on growth rate. 7th World Congress on Genetics Applied to Livestocks Production. Montpellier, August 19-23, 2002. Communication n06-14 (abstract), p 146. 
Abstract

\begin{abstract}
Domestication and genetic improvement of French fish farmed broodstocks in SYSAAF.

Since 1991, French companies have initiated selection of rainbow trout, brown trout, sea bass, sea bream and turbot. The breeders have developed a collective expertise in selection, parental assignement using fingerprints, monosexing, triploidisation, spawning induction, gamete conservation and sperm cryopreservation in the SYSAAF (French union of poultry and aquaculture breeders). This domestication is based on the transfer of the PROSPER selection procedure on growth, developed by INRA (French institute of agricultural research), which is completed by selection on lipid
\end{abstract}

content of the filet and gutted yield, and genealogical reproduction assisted by fingerprints. Genetic improvement of quality is also performed by monosexing and triploidisation. Since 2000, sperm cryopreservation procedure has been adapted for on-farm practice. In 2003 , between 80 to $100 \%$ of the French production of these species, benefits from genetically improved juveniles.

HAFFRAY P., PINCENT C., RAULT P., COUDURIER B., 2004. Domestication et amélioration génétique des cheptels piscicoles français dans le cadre du SYSAAF. INRA Prod. Anim., 17, 243-252. 